\title{
Ethnic differences in health and use of health care: the questions to be answered
}

\author{
Sijmen A. Reijneveld
}

Published online: 7 September 2010

(c) The Author(s) 2010. This article is published with open access at Springerlink.com

Ethnic differences in health and use of health care challenge the international public health highly. This issue of the International Journal of Public Health contains a number of findings on the various mechanisms that contribute to these differences, such as ethnicity in itself and the process and legal basis of the immigration to the hosting country. This editorial summarizes the lessons to be learned from these new findings. It ends with the questions to be answered next. For those, who stop reading editorials after the first paragraph: what we need now is evidence on the pathways leading to ethnic differences in health and on ways to intervene in these pathways. In addition and as part of that the concept of ethnicity and its attributes continue to require scrutiny.

\section{Ethnicity and migration: concept and variation}

First, what is ethnicity, and what is migration? The systematic review of Nielsen and Krasnik (in this issue) provides clear-cut definitions regarding both concepts. They rightly notice that no universal definitions are greed upon, and that the terms that are used to a certain extent reflect the socio-political history in different countries. In their review, they also show the various ways in which these concepts have been measured. For instance, ethnicity has been measured by either country of birth, self-identification or

This paper belongs to the special issue "Health of ethnic minorities in Europe".

S. A. Reijneveld ( $\square)$

Department of Health Sciences, University Medical Center

Groningen, Groningen, The Netherlands

e-mail: s.a.reijneveld@med.umcg.nl citizenship. They did not meet other methods, such as identification by surnames (Spallek et al. 2009), by proxyidentification (van Enk et al. 2000) or by place of settlement (Dostal; Skodova, in this issue). Migration seems to be more clear-cut, moving from one country to another. However, this leaves to be answered what a country actually is, as is shown by the collapse of former Yugoslavia, or in the longer past the Austro-Hungarian monarchy.

\section{Routes from ethnicity and migration to health: not just socioeconomic status}

A second question is why ethnicity and migration should lead to differences in health and use of healthcare. Regarding that it should be noted that most research on ethnic differences in fact focuses on disadvantaged minority groups. Many countries have examples of minority groups that are not disadvantaged and, therefore, usually do not get that much attention from the public health field. For instance, major immigrant groups in the Netherlands concern Germans and Indonesians but these get far less attention than the much smaller group of Dutch Antilleans, the latter having a far more adverse situation (compare Reijneveld 1998).

The focus on disadvantaged groups explains the close connection of the debates on ethnic and on socioeconomic differences in health and use of health care. The available evidence shows that indeed an important part of the poorer health situation of ethnic minorities is due to a lower socioeconomic status (SES), but this is at least partially due to the aforementioned bias in focus. This bears the risk that the contribution of other factors is neglected.

Regarding that, it is a pity that many authors show crude associations of ethnicity and outcomes, and next of analyses 
which have been adjusted for both age, gender and SES. That makes it impossible to separately assess the explanatory power of SES on ethnic differences. In addition, other factors, such as culture, housing conditions, language problems, discrimination, acquaintance with the situation in the host country, and legal status, may add to ethnic difference, as several of the studies in this issue show.

Regarding interventions, this implies that in case of disadvantaged ethnic minorities, evidence on the effectiveness of interventions to reduce socioeconomic differences in health may offer a good starting point. However, other aspects such as cultural sensitivity of healthcare and discrimination should also be considered.

\section{Effects of ethnicity and migration on health}

Several papers in this issue show that ethnicity and migration is associated with a poorer health. Nielsen and Krasnik (in this issue) summarize the evidence on ethnic differences in self-perceived health during the past decade in the 27 countries of the European Union. They rather consistently find that ethnic minority groups perceive their health as poorer, independently of the way in which ethnicity had been measured.

Dostal and co-workers (in this issue) examined the health of Roma and non-Roma children in the Czech Republic, coding all morbidity using a condensed ICD-10 list. They report a two- to sixfold higher occurrence of various infectious diseases among Roma children at ages 0-2 years, but no differences at ages 2-6. In contrast, allergies occurred far less among Roma children, as compared to other children.

Next, three papers show that migration may have adverse effects on health, partially depending on the legal and work status of the immigrant but also on the process that has lead to migration. Sousa et al. (in this issue) show that immigrant workers in Spain are more likely to perceive their health as poor than Spanish-born workers. Interestingly, associations vary by legal status of the migrant, but also by type of contract and by gender. Undocumented immigrants mostly report a poorer self-rated and mental health, in particular males. For documented (legal) immigrants, associations depend on the type of working contract and gender.

Second, Lahana et al. (in this issue) show that Albanian immigrants report a better health status using the SF-36 than native Greeks. However, the immigrants in their study are much younger than the native Greeks, which could explain this. Whether this is indeed the case cannot be derived from their study, and they simultaneously adjust for age and for educational level. After that, differences mostly tend to be small and not statistically significant.
Third, Pikhart et al. (in this issue) report on differences in self-rated health between undocumented and documented immigrants in the Czech Republic. They show that indeed SES explains much of the relatively poorer selfrated health of undocumented immigrants. In this case, age is not much likely to contribute to this because age distributions are much more similar (although not fully).

\section{Effects of ethnicity and migration on use of health care}

The effects of ethnicity and migration on use of health care generally concern two factors that work in opposite directions. On the one hand, the often poorer health of ethnic minority groups leads to higher needs for care. On the other hand, these groups may meet more barriers in the process to get care.

Several papers in this issue show the results of barriers in care on actual use. Norredam et al. (in this issue) find that first-generation immigrants use mental health services to a lesser degree than native Danes. This may be due to the additional barriers that these immigrants meet. In a similar way, first-generation immigrants have previously been shown to contact primary care more frequently than native residents, but to contact specialized care less frequently (Reijneveld 1998). The higher contact rates in primary care may be interpreted as being due to a poorer health, the lower rates in specialized care as the result of the additional barriers that immigrants meet. In addition, Terraza-Nunez et al. (in this issue) show that Ecuadorian immigrants in Spain also meet a number of problems in obtaining care. This shows that for immigrants, language is not the only barrier to be passed.

Second, two papers show that ethnic minorities have a poorer health if reaching care, which may be interpreted as an effect of the barriers that they meet in reaching care. Skodova et al. (in this issue) show that Roma patients are in a poorer psychological state when they reach coronary care, as compared to native Slovaks. Similarly, Dorkova et al. (in this issue) show obstructive sleep apnea (OSA) to be more severe among Roma patients referred for OSA-diagnostic procedures than among other Slovak patients.

Finally, Schoevers et al. (in this issue) assessed the obstacles that female undocumented immigrants in the Netherlands meet in reaching healthcare. They found a number of factors, such as lack of information about patient rights, the way in which the healthcare system works, and professional standards such as confidentiality.

In short, this issue provides an interesting array of evidence and new insights. It also shows the need for further evidence on the pathways leading to ethnic differences in health, including in the role of SES versus other contributing 
factors. This may provide us with cues for interventions as well, partially connected to the general discussion on social determinants of health (WHO Commission on Social Determinants of Health 2008). I wish you much pleasure in further reading.

Open Access This article is distributed under the terms of the Creative Commons Attribution Noncommercial License which permits any noncommercial use, distribution, and reproduction in any medium, provided the original author(s) and source are credited.

\section{References}

Reijneveld SA (1998) Reported health, lifestyles and use of health care of first-generation immigrants in the Netherlands: do socioeconomic factors explain their adverse position? J Epidemiol Commun Health 52:298-304

Spallek J, Arnold M, Hentschel S, Razum O (2009) Cancer incidence rate ratios of Turkish immigrants in Hamburg, Germany: a registry based study. Cancer Epidemiol 33:413-418

van Enk WJ, Gorissen WH, van Enk A (2000) Teenage pregnancy and ethnicity in the Netherlands: frequency and obstetric outcome. Eur J Contracept Reprod Health Care 5:77-84

WHO Commission on Social Determinants of Health (2008) Closing the gap in a generation. Health equity through action ion the social determinants of health. Geneva, WHO 